\title{
Study of Optimization Method for Electromechanical Integration Design
}

\author{
Zhou Fei \\ Xijing University \\ College of Mechanical Engineering \\ Shaanxi Xi'an ,China \\ e-mail: 925884122@qq.com \\ Shang Miao \\ Xijing University \\ College of Mechanical Engineering \\ Shaanxi Xi'an ,China \\ e-mail:445700839@qq.com
}

\begin{abstract}
- the optimization method of electromechanical integration design are many, and including multidisciplinary optimization design has been rapid development. In mechatronics system optimization design, concrete implementation method and application effect that is an important content of mechatronics system optimization design for research of multidisciplinary design optimization method. In this paper, the individual disciplinary feasible is discussed; the collaborative optimization approaches is illustrated; the collaborative optimization method is discussed; and all-in-time analysis optimization has been analyzed; Bi - Level integrated system synthesis is described. Optimization strategy is an important content of multidisciplinary design optimization of a complex system. Optimization strategy plays a vital role for solving the success and efficiency. Optimization strategy often determines the modeling strategy in multidisciplinary design optimization of the whole complex system and code interface of different disciplines model. The optimization methods affect the final result of comprehensive optimization. The research of multidisciplinary design optimization methods is of great significance for promoting the optimization design of the electromechanical integration system and promoting the rapid development of the mechanical and electrical products.
\end{abstract}

Keywords-mechanical and electrical integration; optimization method; collaborative optimization method; discipline is feasible; multidisciplinary feasibility

Electromechanical integration system including mechanical and control subsystem. Mechatronics system involving structural statics, dynamics, control, fluid mechanics, and other disciplines. The traditional design method is difficult to deal with complex connected relationship between disciplines. Multidisciplinary optimization design is a kind of effective design method of mechatronics system design. Said closed relationship between each subsystem and each discipline. The traditional design method is difficult to deal with complex said close relationship between disciplines. Multidisciplinary design optimization is a kind of processing effective design method of hydraulic and electromechanical integration system design. Through years of development, the multidisciplinary design

\author{
Lin Guomin \\ Xijing University \\ College of Mechanical Engineering \\ Shaanxi Xi'an ,China \\ e-mail: 641922530@qq.com \\ Tan Changke \\ Xijing University \\ College of Mechanical Engineering \\ Shaanxi Xi'an ,China \\ e-mail: 995965262@qq.com
}

optimization has formed a single subject and feasible method, multidisciplinary feasible method, the collaborative optimization method, analysis and optimization method, and two integrated system synthesis.

\section{INDIVIDUAL DISCIPLINARY FEASIBLE}

Individual Disciplinary Feasible to retain the feasibility of single subject analysis. Single subject feasible method to use the said variables. Between single discipline feasible method to discipline according to look at as a system optimization design variables. Single subject feasible method to effectively avoid the multidisciplinary analysis optimization [1]. Individual Disciplinary Feasible expression and Multidisciplinary optimization problems of Feasibility optimization problem statement is the same. Subject said between variables is optimized. Single subject frame as shown in Fig .1 and feasible method. Fig .1 shows the two disciplines, $\mathrm{X} \mu=(\mathrm{X} \mu 12, \mathrm{X} \mu, 21), \mathrm{Y}=$

( $\mathrm{Y} 12, \mathrm{Y} 21), \mathrm{U}=(\mathrm{U} 1, \mathrm{U} 2)$.

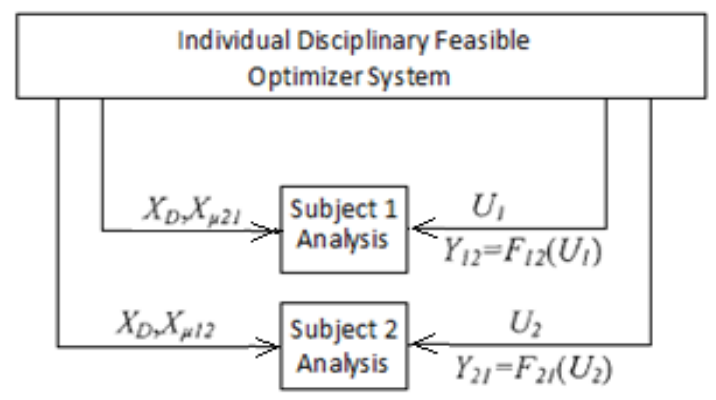

Figure 1. Frame of single discipline feasible method

Among them, $\mathrm{XD}$ as design variables, $\mathrm{X} \mu$ including subject between the variable set, both of design variables set $\mathrm{X}$. Y is subject analysis concluded that the function value; $\mathrm{U}$ are the state variables.

\section{MULTIDISCIPLINARY FEASIBILITY}

Multidisciplinary Feasibility is a kind of used to solve the problem of Multidisciplinary design optimization of the most commonly used method [2]. By the Optimizer (Optimizer) and Multidisciplinary analysis modules, for 
single stage optimization method. Multidisciplinary feasible method of the whole system as a big system, and need to be a necessary relatively concentrated multidisciplinary analysis module. XD design variables are provided by the optimizer, the output state variable U (XD) is obtained after a complete analysis of the multidisciplinary [3]. The XD and $\mathrm{U}$ (XD) is used to calculate the constraint function $\mathrm{g}(\mathrm{XD}, \mathrm{U}(\mathrm{XD}))$ and the objective function $\mathrm{F}$ (XD, U (XD)). Multidisciplinary feasible method framework is shown in Fig . 2.

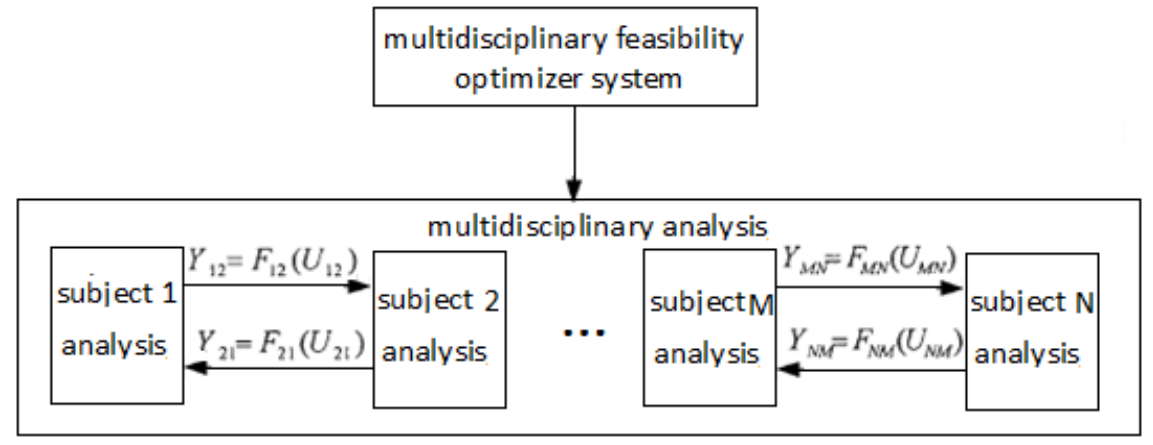

Figure 2. Frame of multidisciplinary feasible method

In the process of multidisciplinary feasible method of optimization, the input variables for optimization. Among the system optimization model, optimization variables only design, at the same time there is no other auxiliary variables [4]. Without adding auxiliary constraint conditions. Compared with other multidisciplinary design optimization method, the optimization model is relatively simple. Every step in the system optimization iteration process, the optimized variables are within the scope of the feasible region. So may be due to the multidisciplinary analysis calculation is too large, lead to optimize the time is too long. Thereby directly interrupt optimization process, although the optimization result is not the optimal solution, but at least is a feasible region scope of feasible solution, also has a certain practical reference value. The shortcoming of multidisciplinary feasible method is a large amount of calculation. Each optimization iteration process must be a complete multidisciplinary analysis. Large amount of calculation are there. In the design of actual engineering products, multidisciplinary feasible method is difficult to be integrated [5]. And because the feasibility of the design point can't be guaranteed. Multidisciplinary feasible method of optimization is also likely to fail.

\section{COLLABORATIVE OPTIMIZATION APPROACHES}

Collaborative Optimization Approaches in 1994 by Kroo puts forward a new kind of pan system Optimization method, the framework is shown in figure 3 [6]. Collaborative optimization method of the optimization problem is divided into two layers of structure, the upper level is to optimize the overall planning system optimizer, the lower is the compatibility optimization of several parallel optimizer discipline level. System level the optimizer will system-level variables optimization of target value assigned to each discipline level optimizer, each discipline level optimization under the precondition of its constraints, the goal function should be called variables and the difference between the target value of the minimum system distribution.

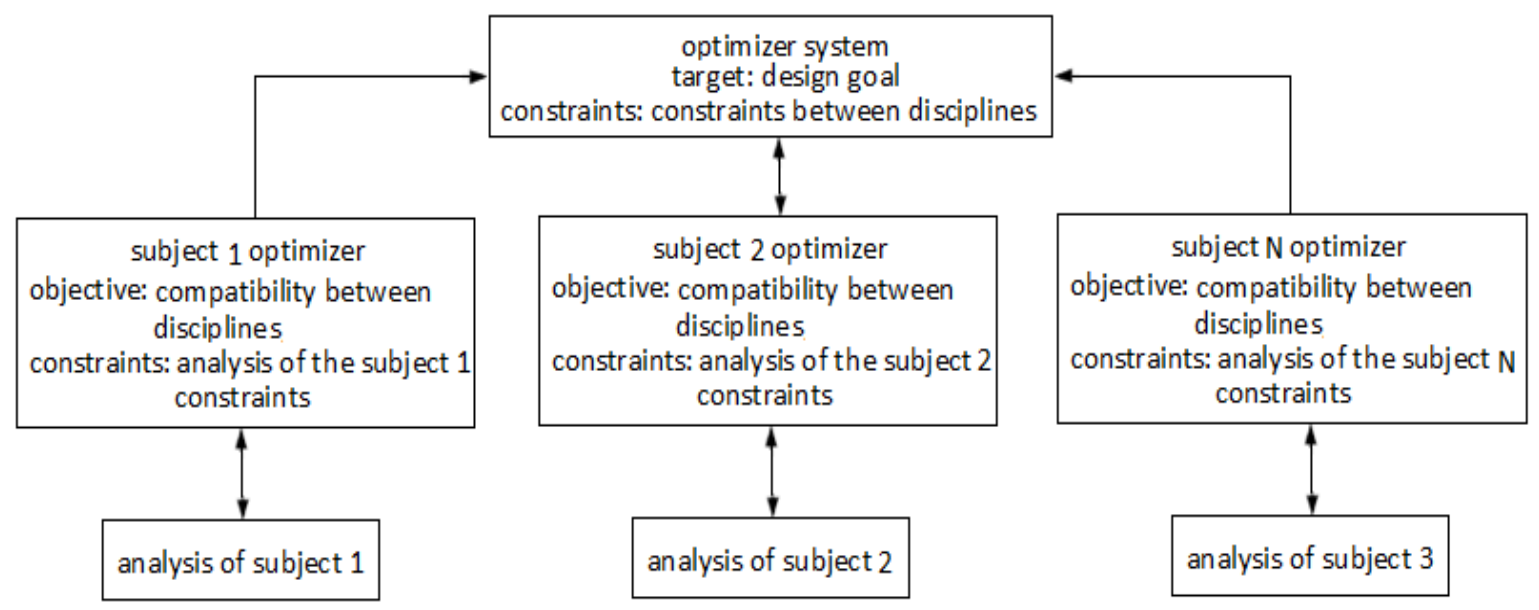

Figure 3. Frame of collaborative optimization method

In All - in - time Analysis Optimization and Individual Disciplinary Feasible method, each subspace (subsystem or disciplines) only for subspace analysis, however, the collaborative optimization method and subspace analysis will have been subsystem and system optimization. Collaborative optimization method, the biggest advantage is to eliminate a lot of system analysis, all subsystem can parallel analysis and optimization [7]. Although not strictly 
prove the convergence of the collaborative optimization method, but the current application instance has been can fully prove the convergence of the collaborative optimization method is very reliable. Although collaborative optimization method has eliminated the system analysis, but its system optimization goal is not involved in the target system. And many of the numerical example results show that the collaborative optimization method can make the subsystem analysis and optimization of the number of times a sharp increase in the total amount of calculation is not reduced.

\section{ALL-IN-TIME ANALYSIS OPTIMIZATION}

All - in - time Analysis Optimization is a method similar to that of the multidisciplinary feasible method. All - in - time Analysis optimization multidisciplinary feasible method and the main difference is to ensure that the design solution to the global feasibility by the optimizer in the process of optimization [8]. All - in - time Analysis Optimization does not need to complete the multidisciplinary Analysis. All - in - time Analysis Optimization does not need to use iterative simulation Analysis to ensure consistency between the disciplines. All - in - time Analysis of Optimization of a feature is the use of instrumental variables methods to remove the coupling relationship between each subject area [9]. Main advantage is that the method can through the parallel analysis way to overcome the order of subject analysis requirements. Use disposable constraints instead of consistency solution of different disciplines. A lot of engineering problems for state variables, and analysis of optimization method is appropriate [10]. Analysis by introducing auxiliary variable optimization method, at the same time to improve the multidisciplinary feasible method requires multidisciplinary analysis of the shortcomings. Between the various disciplines, by introducing auxiliary constraint to ensure consistency of design variables. Each subject is calculated based on the analysis of the main task of the auxiliary constraint conditions of this discipline.

Optimization method and analysis framework is shown in Fig .2. In the analysis and optimization method, the subject need to their subject necessary analysis, thus good autonomy are kept. But due to the introduction of aided design variables and constraints, the original optimization problem becomes more complicated.

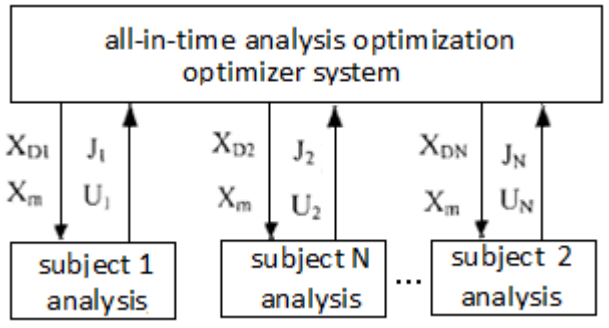

Figure 4. Frame of all-in-time analysis optimization

\section{BI - LEVEL INTEGRATED S YSTEM SYNTHESIS}

Bi - Level Integrated System short is a multidisciplinary optimization design variables are divided into the academic Level of design variables and the System Level design variables for optimization of complex System optimization method. Two stage system synthesis model is shown in Fig .5.

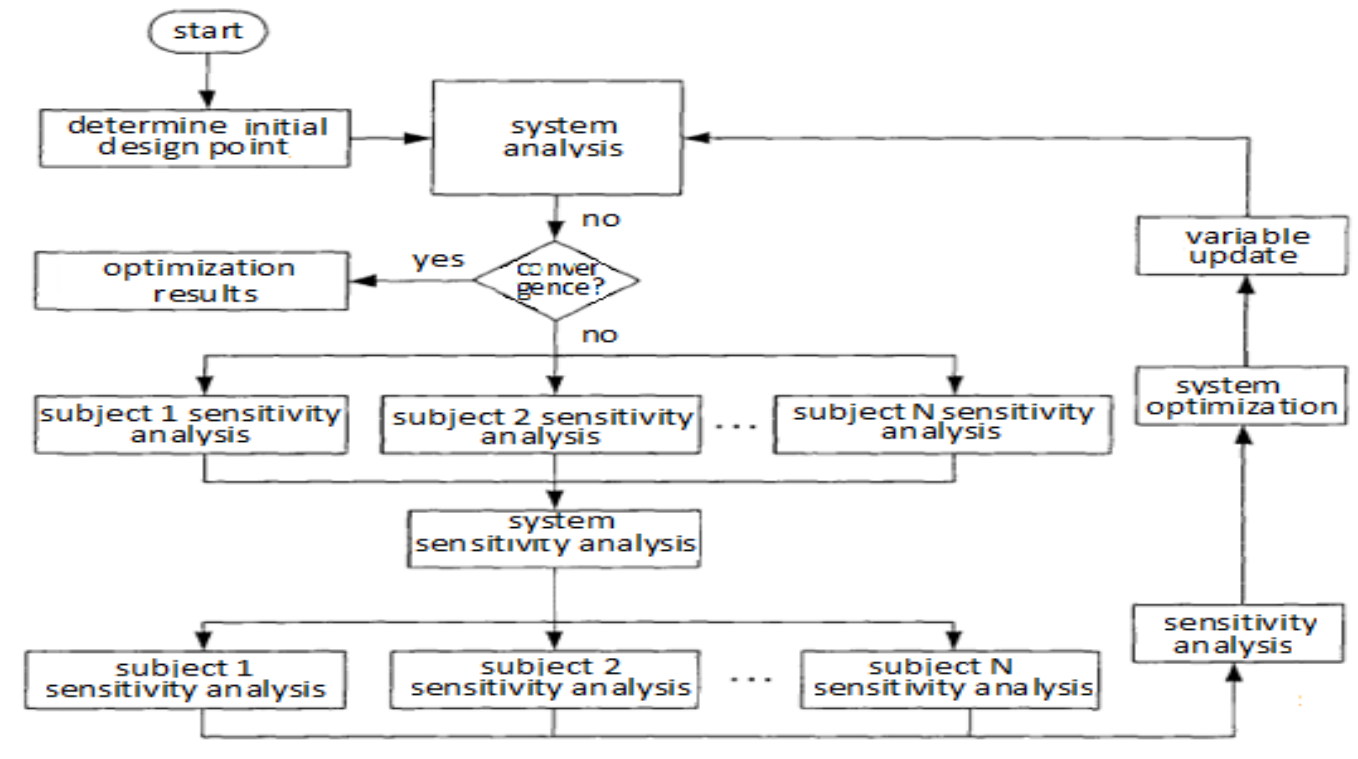

Figure 5. Frame of Bi - Level Integrated System Synthesis

System optimization process began to start running on an initial design point, in the section head, optimize the system level design variables remain the same, discipline level optimizer simply conform to its own constraints can optimize the design variables. Two level synthesis integration system is mainly through the subsystem in the process of optimization to optimize a large number of local variables and through the system optimization process to optimize related to a small number of design variables [11]. In BLISS in the process of optimization, system optimization results through the optimum sensitivity analysis data with the results of optimization subsystem . 
By analyzing and comparing various optimization design method of described earlier, you can clearly see that although the multidisciplinary design optimization framework is different, but reducing the number of subject analysis and optimization calculation, to properly simplify the purpose of the corresponding problem is the same. Due to the two-stage optimization method has its own advantages, so in solving practical engineering problems, has become a priority to use the optimization design method.

\section{CONCLUSIONS}

Mechatronics system involving structural statics, dynamics, control, fluid mechanics, and other disciplines. There is a relation between the subsystems and discipline. The traditional design method is difficult to deal with complex connected relationship between disciplines. Multidisciplinary optimization design is a kind of effective design method of mechatronics system design. Through years of development, the multidisciplinary design optimization has formed a single subject and feasible method, multidisciplinary feasible method, the collaborative optimization method, analysis and optimization method, and two integrated system synthesis. In mechatronics system optimization design, concrete implementation method and application effect are an important content of mechatronics system optimization design. In this paper, the individual disciplinary feasible is discussed; the collaborative optimization approaches is illustrated; the collaborative optimization method is discussed; and all-in-time analysis optimization has been analyzed; $\mathrm{Bi}$ - Level integrated system synthesis is described. Optimization strategy is an important content of multidisciplinary design optimization of a complex system. Optimization strategy plays a vital role for solving the success and efficiency. Optimization strategy often determines the modeling strategy in multidisciplinary design optimization of the whole complex system and code interface of different disciplines model. The optimization methods affect the final result of comprehensive optimization. The research of multidisciplinary design optimization methods is of great significance for promoting the optimization design of the electromechanical integration system and promoting the rapid development of the mechanical and electrical products.

\section{REFERENCES}

[1] Sun Jingmin, Liang Yingchun. Mechanical optimization design [M]. Beijing: mechanical industry publishing house. 2011.

[2] He Xianmei. Intelligent algorithm in multidisciplinary design optimization and the approximate model research [D]. Wuhan: huazhong university of science and technology. 2011.

[3] Xiao Mi. Approximate in multidisciplinary design optimization model and solution strategy research [D]. Wuhan: huazhong university of science and technology. 2012.

[4] Su Zijian. Multidisciplinary design optimization of decomposition, synergy and uncertainty study [D]. Wuhan: huazhong university of science and technology. 2008.

[5] Nie Yongjun. Multidisciplinary design optimization technology and its application [J]. Mechanical and electrical product development and innovation, 2011.24 (1) : 4-6.

[6] Gao Qin and Long Yong, chang-lin ma, wen-liang guan. Mechanical and electrical integration of liquid system modeling and simulation technology [M]. Beijing: electronic industry press. 2012.

[7] li-xun zhang. Mechanical and electrical system modeling and simulation [M]. Harbin: Harbin industrial university press. 2010.

[8] MeiXiaoNing shu-xing Yang. Review the multidisciplinary design optimization of complex systems $[\mathrm{J}]$. Journal of engineering design, 2010, (3) : 173-180.

[9] JiGuoHua, li yan, wen-qiang li, etc. A multidisciplinary design optimization method for complex system [J]. Journal of mechanical design and research of 2011.27 (4) : 6-11.

[10] Xue Lipeng. Tilt rotor machine pneumatic/dynamics multidisciplinary design optimization research [D]. Nanjing: nanjing university of aeronautics and astronautics. 2011.

[11] JiaoLiMing. Multidisciplinary design optimization theory in the application of parallel mechanism [D]. Zhengzhou: zhengzhou university. 2012. 